\title{
Erratum to: IROA: International Register of Open Abdomen, preliminary results
}

Federico Coccolini ${ }^{1 *}$, Giulia Montori ${ }^{1}$, Marco Ceresoli ${ }^{1}$, Fausto Catena ${ }^{2}$, Rao Ivatury ${ }^{3}$, Michael Sugrue ${ }^{4}$ Massimo Sartelli ${ }^{5}$, Paola Fugazzola ${ }^{1}$, Davide Corbella ${ }^{6}$, Francesco Salvetti ${ }^{1}$, lonut Negoi ${ }^{7}$, Monica Zese ${ }^{8}$, Savino Occhionorelli ${ }^{8}$, Stefano Maccatrozzo ${ }^{8}$, Sergei Shlyapnikov ${ }^{9}$, Christian Galatioto ${ }^{10}$, Massimo Chiarugi ${ }^{10}$, Zaza Demetrashvilii ${ }^{11}$, Daniele Dondossola ${ }^{12}$, Yovcho Yovtchev ${ }^{13}$, Orestis Ioannidis ${ }^{14}$, Giuseppe Novelli ${ }^{15}$, Mirco Nacoti ${ }^{16}$, Desmond Khor ${ }^{17}$, Kenji Inaba ${ }^{17}$, Demetrios Demetriades ${ }^{17}$, Torsten Kaussen ${ }^{18}$, Asri Che Jusoh ${ }^{19}$, Wagih Ghannam ${ }^{20}$, Boris Sakakushev ${ }^{21}$, Ohad Guetta ${ }^{22}$, Agron Dogjani ${ }^{23}$, Stefano Costa ${ }^{24}$, Sandeep Singh ${ }^{25}$, Dimitrios Damaskos ${ }^{26}$, Arda Isik ${ }^{27}$, Kuo-Ching Yuan ${ }^{28}$, Francesco Trotta ${ }^{29}$, Stefano Rausei ${ }^{30}$, Aleix Martinez-Perez ${ }^{31}$, Giovanni Bellanova ${ }^{32}$, Vinicius Cordeiro Fonseca ${ }^{33}$, Fernando Hernández ${ }^{34}$, Athanasios Marinis ${ }^{35}$, Wellington Fernandes ${ }^{36}$, Martha Quiodettis ${ }^{37}$, Miklosh Bala ${ }^{38}$, Andras Vereczkei ${ }^{39}$, Rafael L. Curado ${ }^{40}$, Gustavo Pereira Fraga ${ }^{40}$, Bruno M. Pereira ${ }^{40}$, Mahir Gachabayov ${ }^{41}$, Guillermo Perez Chagerben ${ }^{42}$, Miguel Leon Arellano ${ }^{43}$, Sefa Ozyazici ${ }^{44}$, Gianluca Costa ${ }^{45}$, Tugan Tezcaner $^{46}$ and Luca Ansaloni ${ }^{1}$

\section{Erratum}

The original article [1] mistakenly incorporated a sentence within the Abstract that stated:

"Mean BMI: 36 +/- 5.6"

This statement was incorrect and has now been removed from the original article.

\begin{abstract}
Author details
'General, Emergency and Trauma Surgery Department, Papa Giovanni XXIII Hospital, Piazza OMS 1, 24127 Bergamo, Italy. ${ }^{2}$ Emergency Surgery Department, Parma University Hospital, Parma, Italy. ${ }^{3}$ Virginia Commonwealth University, Richmond, VA, USA. ${ }^{4}$ General Surgery, Letterkenny Hospital, Donegal, Ireland. ${ }^{5}$ General and Emergency Surgery Department, Macerata Hospital, Macerata, Italy. ${ }^{6}$ Neuro Intensive Care Unit Department, Papa Giovanni XXIII Hospital, Bergamo, Italy. ${ }^{7}$ Emergency Surgery Hospital, Bucharest, Romania. ${ }^{8}$ Emergency Surgery Department, Ferrara University Hospital, Ferrara, Italy. ${ }^{9}$ Science Research of Emergency Care N. A., Djanelidze, Russia. ${ }^{10}$ Azienda Ospedaliera Universitaria Pisana, Pisa, Italy. ${ }^{11}$ Kipshidze Central University Hospital, Kipshidze, Georgia. ${ }^{12}$ HPB Surgery, Fondazione IRCCS Cà Granda Ospedale Maggiore Policlinico, Milano, Italy. ${ }^{13}$ University Hospital "Prof Stoian Kirkovich" AD, Stara Zagora, Bulgaria. ${ }^{14}$ Fourth Surgical Department, Hospital George Papanikolau, Aristotle University, Thessaloniki, Greece. ${ }^{15}$ General Surgery, Infermi Hospital, Rimini, Italy. ${ }^{16}$ Pediatric Intensive Care Unit, Papa Giovanni XXIII Hospital, Bergamo, Italy. ${ }^{17}$ LAS + USC Medical Centre, Los Angeles, California, USA. ${ }^{18}$ Pediatric Intensive Care Unit, Hannover University Hospital, Hannover, Germany. ${ }^{19}$ Khuala Krai Hospital, Kuala Krai, Malaysia. ${ }^{20}$ Mansoura Faculty of Medicine, Mansoura, Egypt. ${ }^{21}$ Medical University of Plovdiv, Plovdiv, Bulgaria. ${ }^{22}$ Soroka Medical Centre, Beersheba, Israel. ${ }^{23}$ University Hospital of Trauma, Tirana, Albania. ${ }^{24}$ Emergency and General Surgery, Fondazione IRCCS Cà Granda Ospedale Maggiore Policlinico, Milano, Italy. ${ }^{25}$ Oxford University Hospital, Oxford, UK. ${ }^{26}$ John Radcliffe Hospital, Oxford, UK. ${ }^{27}$ Erzincan University Faculty of Medicine Mengucek Gazi Training Research Hospital Erzincan, Erzincan, Turkey.
\end{abstract}

${ }^{28}$ Chang Gung Memorial Hospital, Taoyuan, Taiwan. ${ }^{29}$ Ospedale Maggiore, Lodi, Italy. ${ }^{30}$ Ospedale di Circolo e Fondazione Macchi, University of Insubria, Varese, Italy. ${ }^{31}$ Hospital Universitario Doctor Peset, Valencia, Spain. ${ }^{32}$ S.S. Annunziata Hospital, Taranto, Italy. ${ }^{33}$ Hospital Santa Virgínia, São Paulo, Brazil. ${ }^{34} \mathrm{H}$ ospital Central Militar, Mexico City, Mexico. ${ }^{35}$ Tzaneio General Hospital of Piraeus, Piraeus, Greece. ${ }^{36}$ Hospital Regional de Sao Jose, San Jose, Brazil. ${ }^{37}$ Hospital Santo Tomás, Panama City, Panama. ${ }^{38}$ Hadassah Hebrew University Medical Center, Jerusalem, Israel. ${ }^{39}$ Department of Surgery, Medical School University Pécs, Pécs, Hungary. ${ }^{40}$ Hospital De Clinicas Da Unicamp, Campinas, Brazil. ${ }^{41}$ Vladimir City Clinical Hospital of Emergency Medicine, Vladimir City, Russia. ${ }^{42}$ University Hospital, Cuenca, Ecuador. ${ }^{43}$ Hospital La Paz, Madrid, Spain. ${ }^{44}$ Adana Numune Training and Research Hospital, Department of Surgery, Adana, Turkey. ${ }^{45}$ Ospedale Sant' Andrea University Hospital Sapienza, Rome, Italy. ${ }^{46}$ Baskent University School of Medicine, Ankara, Turkey.

Received: 3 March 2017 Accepted: 6 March 2017

Published online: 09 March 2017

\section{Reference}

1. Coccolini F, et al. IROA: International Register of Open Abdomen, preliminary results. World J Emerg Surg. 2017;12:10.

\footnotetext{
* Correspondence: federico.coccolini@gmail.com

'General, Emergency and Trauma Surgery Department, Papa Giovanni XXIII Hospital, Piazza OMS 1, 24127 Bergamo, Italy
} 